\title{
Cholinergic enhancement modulates neural correlates of selective attention and emotional processing
}

\author{
Paul Bentley, ${ }^{\mathrm{a}, *}$ Patrik Vuilleumier, ${ }^{\mathrm{b}}$ Christiane M. Thiel, ${ }^{\mathrm{a}, \mathrm{c}}$ \\ Jon Driver, ${ }^{\mathrm{b}}$ and Raymond J. Dolan ${ }^{\mathrm{a}}$ \\ ${ }^{a}$ Wellcome Department of Imaging Neuroscience, Institute of Neurology, 12 Queen Square, London WCIN 3BG, UK \\ ${ }^{\mathrm{b}}$ Institute of Cognitive Neuroscience, 17 Queen Square, London WCIN 3AR, UK \\ c Institut für Medizm (IME), Forschungszentrum Jülich, 52425 Juelich, Germany
}

Received 23 December 2002; revised 17 April 2003; accepted 1 May 2003

\begin{abstract}
Neocortical cholinergic afferents are proposed to influence both selective attention and emotional processing. In a study of healthy adults we used event-related fMRI while orthogonally manipulating attention and emotionality to examine regions showing effects of cholinergic modulation by the anticholinesterase physostigmine. Either face or house pictures appeared at task-relevant locations, with the alternative picture type at irrelevant locations. Faces had either neutral or fearful expressions. Physostigmine increased relative activity within the anterior fusiform gyrus for faces at attended, versus unattended, locations, but decreased relative activity within the posterolateral occipital cortex for houses in attended, versus unattended, locations. A similar pattern of regional differences in the effect of physostigmine on cue-evoked responses was also present in the absence of stimuli. Cholinergic enhancement augmented the relative neuronal response within the middle fusiform gyrus to fearful faces, whether at attended or unattended locations. By contrast, physostigmine influenced responses in the orbitofrontal, intraparietal and cingulate cortices to fearful faces when faces occupied task-irrelevant locations. These findings suggest that acetylcholine may modulate both selective attention and emotional processes through independent, region-specific effects within the extrastriate cortex. Furthermore, cholinergic inputs to the frontoparietal cortex may influence the allocation of attention to emotional information.
\end{abstract}

(C) 2003 Elsevier Inc. All rights reserved.

Keywords: fMRI; Cholinergic; Physostigmine; Attention; Emotion

\section{Introduction}

Numerous lines of evidence indicate that corticopetal cholinergic projections originating in nucleus basalis may modulate attention, through influences both on a frontoparietal network thought to mediate "top-down" control and on sensory cortices subserving "bottom-up" stimulus processing (Sarter et al., 2001; Muir et al., 1996; Robbins, 1998). Cholinergic inputs to frontoparietal cortex have been associated with performance on sustained and selective attention tasks (McGaughy et al., 1996; Himmelheber et al.,

\footnotetext{
* Corresponding author. Wellcome Dept. of Imaging Neuroscience, 12 Queen Square, London WCIN 3 BG, UK. Fax: +020-7813-1420.

E-mail address: p.bentley@fil.ion.ucl.ac.uk (P. Bentley).
}

2001), with attention-correlated-neural activity (Gill et al., 2000), and covert attentional shifts (Davidson and Marrocco, 2000). On the other hand, cholinergic neurotransmission within primary and secondary sensory cortices can facilitate stimulus processing via positive effects on signalto-noise ratio (Sato et al., 1987), information flow (Hasselmo, 1995; Xiang et al., 1998), and response selectivity (Murphy and Sillito, 1991). Cholinergic influences on attention are also suggested by the fact that attentional deficits found in dementias associated with cholinergic degeneration (Perry and Hodges, 1999; Ballard et al., 2001) are more amenable to correction with anticholinesterases than other cognitive deficits (Lawrence and Sahakian, 1995; McKeith et al., 2000). A recent fMRI study (Furey et al., 2000a) suggested that effects of acetylcholine on stimulus-process- 
ing regions might occur selectively during particular stages of a task e.g., encoding into working memory. It has not yet been shown, however, that cholinergic manipulation can modify activity in sensory cortices specifically attributable to attention, rather than to concomitant changes in stimulus or task (see also Lawrence et al., 2002).

Other research suggests a role for neocortical cholinergic modulation in emotional processing (e.g., conditioning, fear responses, anxiety). Cholinergic inputs have been shown in rodents to facilitate conditioning via effects on sensory cortices (Weinberger et al., 1998; Delacour et al., 1990), while cholinergic blockade has recently been shown with a human fMRI study to inhibit conditioning-related responses in auditory cortex (Thiel et al., 2002a). Such data complement anatomical (Kapp et al., 1990; Amaral et al., 1992), neurophysiological (Weinberger et al., 1990; Wilson and Rolls, 1990), and computational (Friston et al., 1994) studies suggesting a role for nucleus basalis cholinergic fibres in relaying evaluative processing within regions such as the amygdala to selection and learning mechanisms in the thalamus and cortex. Increased cholinergic tone in the prefrontal cortex may also be expressed for behaviorally, significant or novel stimuli (Acquas et al., 1996; Pirch et al., 1992; Wilson and Rolls, 1990), which when continually hyperactive may engender clinical anxiety (Bernston et al., 1998; Hart et al., 1999). These findings suggest that cholinergic afferents to specific sensory and prefrontal regions may contribute to "automatic" enhancement of emotional stimulus processing, independently of whether such stimuli are attended.

The present study crossed factors of selective attention and emotion within a fully orthogonal design to examine modulation of condition-specific fMRI responses by cholinergic enhancement with the centrally acting anticholinesterase physostigmine. The paradigm was similar to that of recent functional MRI studies with untreated human subjects, in which the response of the fusiform gyrus to faces was found to be increased by both selective spatial attention and emotional expression (Wojciulik et al., 1998; Vuilleumier et al., 2001). Since acetylcholine has been found in animals to benefit selective attention and emotional processing, we predicted that the differential response of the fusiform cortex to both factors would be independently enhanced with physostigmine. We also assessed whether regions of the extrastriate cortex preferentially activated for attending houses versus faces-parahippocampal and posterolateral occipital cortices (Vuilleumier et al., 2001) might also show a greater differential effect under physostigmine. Finally, as cholinergic afferents to parietal (Holland and Gallagher, 1999) and orbitofrontal (Cavada et al., 2000; Aou et al., 1983) cortices have been proposed to mediate attentional recruitment by emotional stimuli, we predicted that these regions would show modulation by physostigmine specifically when emotional faces were task- irrelevant. On the other hand, task-relevant emotional stimuli, being already attended, would not be expected to engage this cholinergic facilitated circuit.

\section{Materials and methods}

\section{Subjects}

Thirty healthy right-handed volunteers with no history of medical or psychiatric disease gave written informed consent. They were divided into two groups of 15 (placebo, 7 female, 8 male; mean age, $26.8 \pm 2.3$; physostigmine, 8 female, 7 male; mean age, $23.5 \pm 2.0$ ). No subject was taking active medication. While 2 subjects were mild smokers, they were in different groups and refrained from smoking on the test day. A between-subjects design was chosen for the pharmacological manipulation, to avoid habituation effects that may occur in within-subjects designs following repeated exposure to emotional stimuli (Breiter et al., 1996).

\section{Drug treatment}

A double-blind placebo-controlled drug administration technique was used. Each subject received an intravenous cannula into the left cubital fossa and an infusion of either physostigmine or saline. Dosage and rate of physostigmine infused were identical to those used in a recent study (Furey et al., 2000b), which demonstrated significant and stable levels of plasma drug concentration and butyrylcholinesterase inhibition, as well as a significant and stable effect on cognitive performance for $40 \mathrm{~min}$, following a 40-min loading period. The same protocol has also been found to result in changes in both regional cerebral blood flow (rCBF) and blood oxygenation level-dependent (BOLD) activity, during visual working memory tasks (Furey et al., 1997, 2000a).

Subjects in the drug group first received $0.2 \mathrm{mg}$ of intravenous glycopyrrolate-a peripheral muscarinic receptor antagonist - to reduce peripheral side effects. The placebo group were injected with an equivalent volume of saline. Both groups then received an intravenous infusion. For the drug group this consisted of physostigmine at a rate of $1.93 \mathrm{mg} /$ hour for $10 \mathrm{~min}$, followed by a constant rate of $0.816 \mathrm{mg} /$ hour for $40 \mathrm{~min}$, before scanning. The same rate was then continued until the end of study so that each subject received no more than $1.3 \mathrm{mg}$ of physostigmine in total. The placebo group received an equivalent volume of saline over the same time course. Both groups of subjects had their blood pressure checked before and at $40 \mathrm{~min}$ into infusion; pulse oximetry was performed continuously throughout the experiment. Subjects were also given questionnaires at these two time points to document side effects and subjective ratings (Bond and Lader, 1974). 


\section{Cognitive task}

Subjects performed a matching task (Vuilleumier et al., 2001) for two black and white photographs situated in either the north-south or east-west positions of a cross-format display that comprised four concurrent photographs $\left(3^{\circ} \times\right.$ $5^{\circ}$ visual angle each), arranged into a cross around a central fixation point (Fig. 1). At the start of each block, subjects were cued (for $2 \mathrm{~s}$ ) to attend selectively to either the two vertically arranged or two horizontally arranged positions, while the alternative two locations were to be ignored throughout the block. In total, there were four blocks of 40 trials each. Each trial consisted of a central fixation cross (1 s) followed by the four-picture display for $250 \mathrm{~ms}$. Subjects were required to indicate, as accurately and rapidly as possible, whether the two stimuli at task-relevant locations were the same or different, by either of two possible key presses with the right hand. Reaction time (RT) and accuracy were recorded.

Within each trial, either the two attended or the two unattended locations were occupied by two faces, in an intermingled and unpredictable sequence. The remaining two locations were occupied by two houses. Hence each trial could be classified as faces-attended (A) or facesunattended (U) in this sense (with the type of attended stimulus thus being determined by spatial location). Furthermore, faces could have either a fearful emotion (E) or a neutral $(\mathrm{N})$ expression, independently of whether they were at task-relevant locations. Thus four conditions existed. AE, $\mathrm{AN}$, UE, and UN (where AE, for example, would represent trials where fearful faces appeared at attended task-relevant locations). The four trial types, and pair identities (i.e., same/different, which was independent between the attended and unattended pair in each trial), were randomly counterbalanced throughout each block. The order of taskrelevant locations (i.e., either vertical or horizontal) between blocks was randomly selected from one of four alternatives (HVHV, VHVH, HVVH, VHHV) and counterbalanced across subjects within each group.

Although the task design was identical to that used in our recent study of untreated subjects (Vuilleumier et al., 2001), there were four differences in details: (1) the median intertrial interval $(2.5 \mathrm{~s}$; range, $1.5-14.4 \mathrm{~s})$ was half that used previously; (2) the number of trials of each type was reduced from 52 to 40 ; (3) an alternative set of pictoral stimuli were used (faces taken from The Karolinska Directed Emotional Faces set; Lundqvist et al., 1998), with each being repeated only once; (4) 40 "null" trials were included in which a blank screen occurred, following a 1-s central fixation cross (thus enabling measurement of any attentional activity in the absence of stimulation: see Chawla et al., 1999). The first two changes were implemented because of time constraints imposed by drug administration.

The cross-format spatial array and brief exposure time have previously been shown to be effective at engaging covert attention to the relevant pair of locations without saccades (Vuilleumier et al., 2001; Wojciulik et al., 1998), as well as enabling emotional processing without awareness of unattended fearful faces (Vuilleumier et al., 2001). We nevertheless monitored eye movements throughout the task with an infrared eye tracker (ASL Model 540, Applied Science Group Co., Bedford, MA; refresh rate, $60 \mathrm{~Hz}$ ). For technical reasons, eye-position data were lost for six subjects (two placebo and four from drug group).

\section{Imaging and image processing}

MRI data were acquired from a 2-T VISION system (Siemens, Erlangen, Germany) equipped with a head coil. Functional images were acquired with a gradient echoplanar T2* sequence using BOLD contrast. The acquired image consisted of $32 \times 3 \mathrm{~mm}$ thickness axial slices that covered the entire brain. Volumes were acquired in a single continuous session with an effective repetition time of 3.26. The first eight volumes were discarded, to allow for $\mathrm{T} 1$ equilibration effects. Images were realigned, time corrected, normalized to a standard echo-planar image template, and smoothed with a Gaussian kernel of $8 \mathrm{~mm}$ full-width halfmaximum.

\section{Statistical analysis of images}

Data were analyzed with a general linear model for event-related designs (SPM99; Wellcome Dept. of Cognitive Neurology, London, UK; Friston et al., 1995) using a random effects analysis. Data were globally scaled and high-pass filtered at $1 / 120 \mathrm{~Hz}$. Individual events were modeled by a canonical synthetic hemodynamic response function and its temporal derivative, aligned with the onset of the picture array. Time-related changes specific to each event type were included using a linear trend model, after being orthogonalized with respect to time-constant effects (Buchel et al., 1999). The six head movement parameters were included as confounds, and incorrect responses were modeled separately. Since face stimuli were presented twice, and repetition effects may themselves be cholinergically modulated (Thiel et al., 2002b), a second model was generated in which repetition effects were included as a separate factor. None of the drug-by-condition interactions presented here could be accounted for by repetition effects (see Bentley et al., 2003).

Linear contrasts of parameter estimates for each subject were used to generate statistical parametric maps (SPMs) of the $t$ statistic. We first examined regions specific to attended stimulus type (i.e., attending faces minus attending houses or vice- versa) in the placebo and physostigmine groups separately. We next performed $t$-tests that directly compared drug and placebo groups for the same contrasts across the whole brain. Similarly, we identified regions activated by emotional versus neutral faces (independent of attention) in each group separately, before comparing drug and placebo groups for this. For all drug $\times$ condition interactions, 


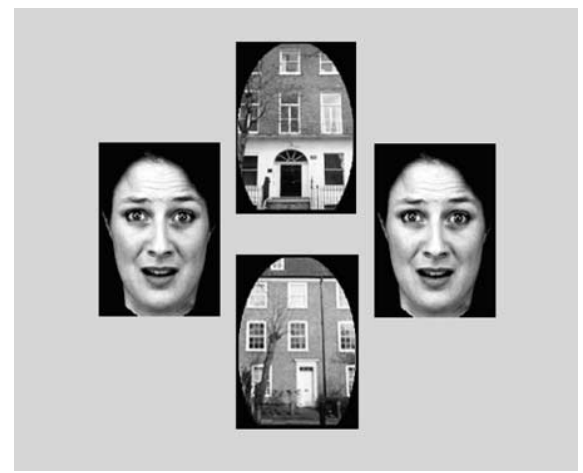

Fig. 1. A stimulus example is shown. Before each block the subject was cued to attend either the two horizontal or the two vertical locations via a pair of highlighted frames. During the block subjects were required to perform a same/different judgment for the pair of stimuli at just the task-relevant locations; the other pair of stimuli were task-irrelevant. Each display contained one pair of faces and one pair of houses, with either type equally likely to be at the relevant or irrelevant locations, in an unpredictable sequence. The pair of faces could both be fearful (emotional trial) or both neutral.

only regions showing a significant effect of face or house attention, or of emotion $(P<0.001$, uncorrected $)$, in either group are noted. Finally, to characterize the nature of any three-way interactions of attention, emotion, and drug, we performed post hoc ANOVAs on signal estimates of drug $\mathrm{x}$ emotion interactions, separately for trials with faces relevant versus irrelevant. Results are listed according to which of the two levels of attention showed a significant drug $X$ emotion interaction $(P<0.05)$; regions in which a signif- icant interaction occurred under both levels of face attention are noted separately.

Since one major issue concerned any cholinergic modulation of fusiform face-responsive areas in the present paradigm, we derived two regions of interest (ROI) from the bilateral fusiform areas identified from our previous study, which had demonstrated attentional modulation to faces in untreated subjects using a similar paradigm (thresholded at $P<0.05$, uncorrected; Vuilleumier et al., 2001). We report areas that achieved significance after correction either within these prespecified ROIs (Worsley et al., 1996) or for the entire brain volume, plus activations that reached $P<$ 0.001 , uncorrected.

\section{Results}

Physical data, subjective reports, and eye tracking

Questionnaires detailing possible side effects and subjective feelings as well as measures of blood pressure and pulse were recorded before infusion and just prior to scanner entrance, when a steady state of physostigmine would be expected (Furey et al., 2000b). Although subjects given physostigmine with glycopyrrolate were more likely to experience a dry mouth $(\mathrm{U}=62, P<0.05)$ and dizziness ( $\mathrm{U}$ $=68, P<0.01)$, the mean intensity of these symptoms was small $(1.3 \pm 0.95$ and $0.5 \pm 0.40$, respectively, on a scale of 0 to 6$)$. Two subjects given physostigmine who vomited were excluded and replaced with alternative subjects. A
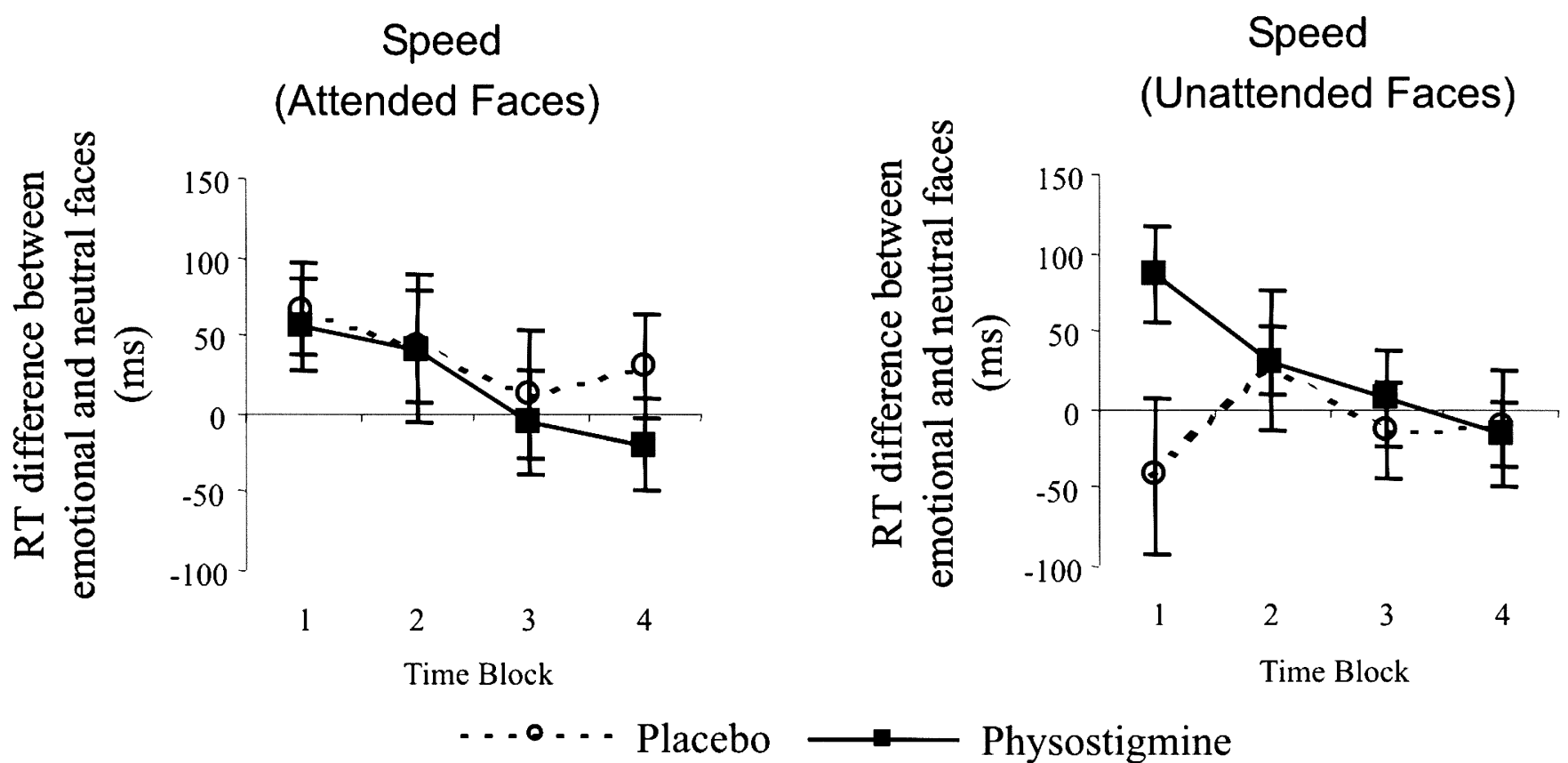

Fig. 2. Plots show differences in RT (in milliseconds) between emotional minus neutral trials over block number for placebo and physostigmine groups separately for attended (A) and unattended (B) faces. An asterisk denotes significant between-group difference $(P<0.05)$ for the planned comparison in the first block when emotional effects would be expected to be greatest. Bars denote standard errors. 
Face-Selective Areas

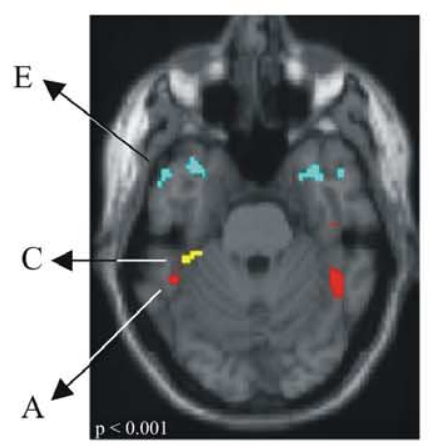

House-Selective Areas
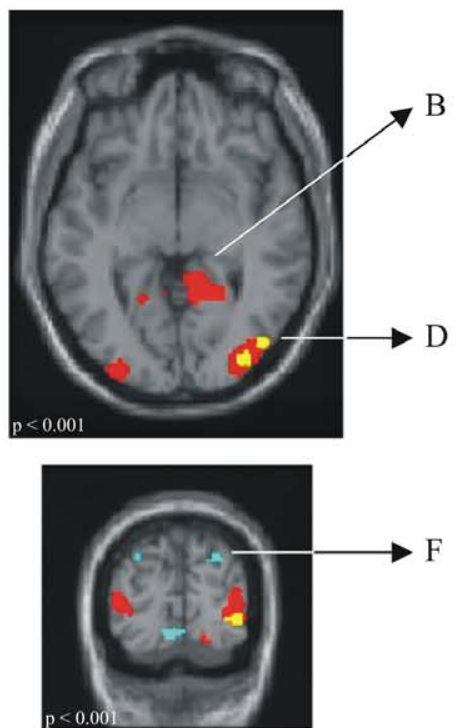

Face-Selective Areas

House-Selective Areas

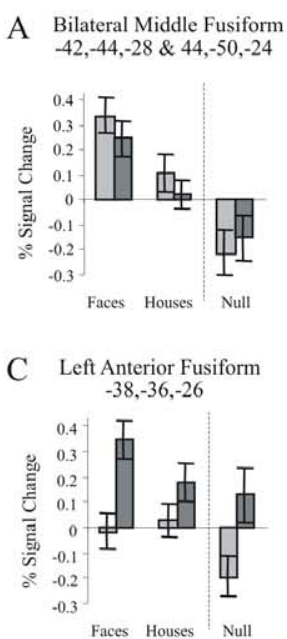

E Bilateral Temporal Poles $38,2,-42 \&-56,-2,-32$

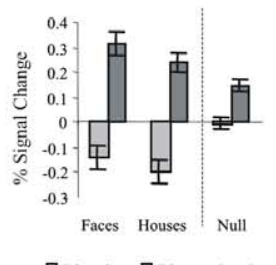

B Bilat. Parahippocampal $16,-52,6 \&-24,-36,-14$

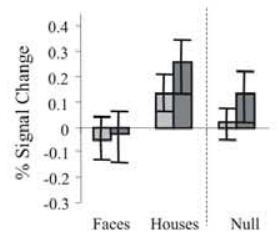

Right Lateral Occipital
$38,-88,4$

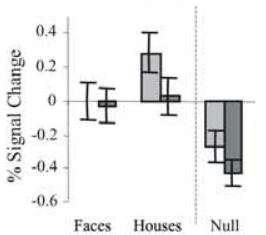

F Right Sup. Occipital

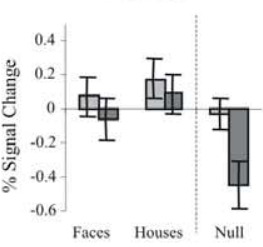

口Placebo $\square$ Physostigmine

Fig. 3. Regions of inferotemporal cortex showing differing effects of physostigmine on attentional processing in face (A,C,E) and house-selective regions (B,D,F). Red represents regions that in the placebo group showed an increased response to faces (A) or to houses (B) in attended versus unattended locations; the physostigmine group can be seen to display similar effects in both fusiform and parahippocampal areas. Yellow represents regions in which physostigmine, relative to placebo, modulated the attentional effect by either increasing the differential response to faces in attended versus unattended locations (C) or decreasing the differential response to houses in attended versus unattended locations (D). Cyan represents regions in which physostigmine, relative to placebo, resulted in increased (E) or decreased (F) responses to null trials, i.e., when subjects were cued, but no stimulus appeared. All contrasts are thresholded at $P<0.001$, uncorrected. Regions in $\mathrm{E}$ and $\mathrm{F}$ also showed selectivity for face and house attention, respectively, over both groups $(P<0.01$, uncorrected). Activation maps are superimposed on a single-subject T1-weighted MRI brain, pitched to visualize all relevant contrasts. Graphs show percentages of signal change from baseline with faces in attended locations, houses in attended locations, and null trials for drug and placebo groups, mean-corrected between regions.

pooled measure of subjective alertness (Bond and Lader, 1974) suggested that the physostigmine group felt more drowsy at test relative to preinfusion (mean percentages of difference between preinfusion and prescan, $-1.3 \pm 2.2 \%$ for placebo and $+8.8 \pm 3.7 \%$ for physostigmine; $F(1,28)=$ 6.4; $P<0.05$ ), although the simple effects of group for absolute subjective alertness at each time point were insignificant. There were no significant cardiovascular main effects or interactions.

The frequency of saccades and median angular deviation of the eye were measured during 250-ms epochs before and after the onset of each stimulus. These measurements were entered into a three-way ANOVA with factors of group, attention, and emotion. The mean percentages of trials with saccades over both epochs were 3.2 and 3.0 under placebo and physostigmine, respectively. There were no reliable group differences during either of the two peristimulus epochs, either as a main effect or as an interaction with condition, for either saccade number or median ocular position. Finally, the same two measurements were compared within the first block only, given some group differences found in task performance for this block (see below). Once again, no significant main effects or interactions with condition were found.

\section{Task performance}

A nonsignificant trend for faster responses was evident with physostigmine (mean RTs, $844 \pm 62 \mathrm{~ms}, 915 \pm 62 \mathrm{~ms}$, under drug and placebo, respectively; $t(28)=1.4 ; P<0.1$, one-tailed based on Furey at al., 1997), but there was no difference in accuracy between groups (mean scores, $85 \pm$ $3.2 \%$ and $83 \pm 3.2 \%$, under drug and placebo, respectively; $t(28)=-0.7$; ns; two-tailed hereon). There were no significant effects of group as a function of condition (attention, emotion, or their interaction) for either measure. 

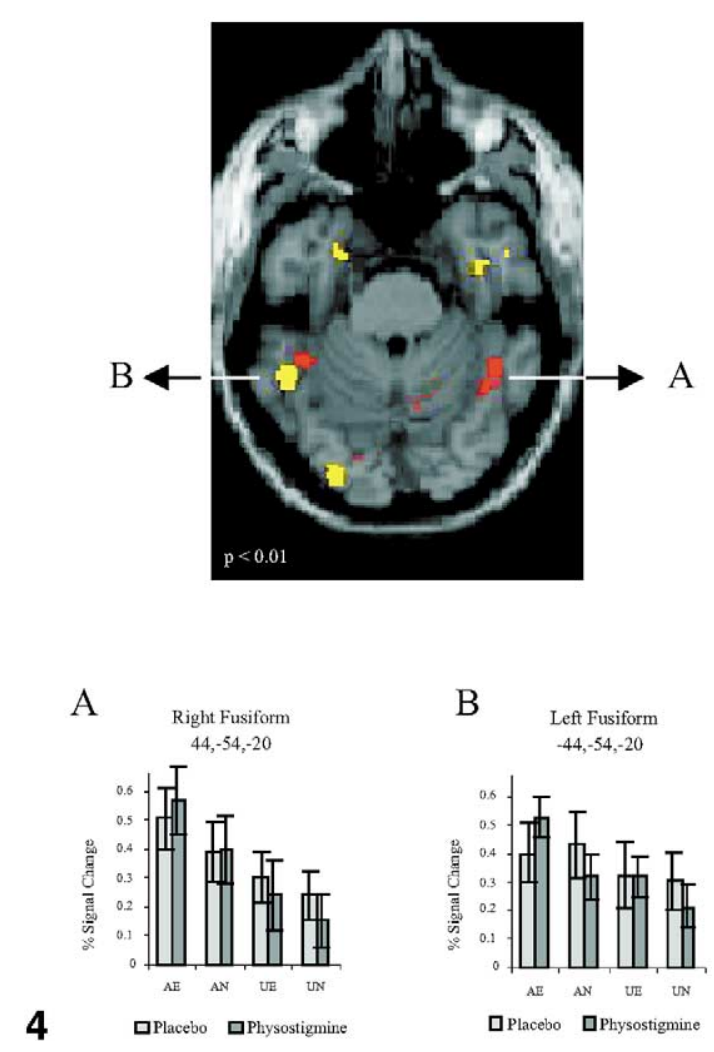

A

B

Bilateral Orbitofrontal Cortex
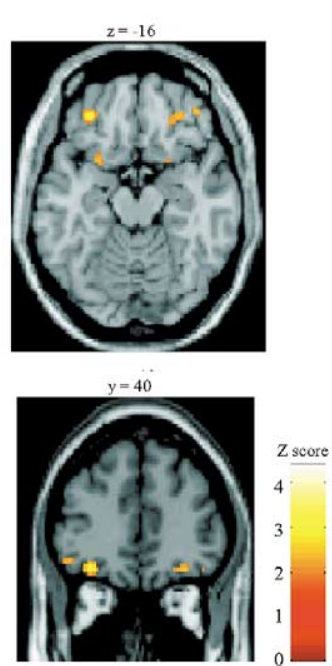

Left Lateral OFC

$-30,40,-16$

「๋

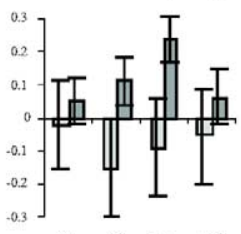

5 口Placebo $\square_{\text {Physostigmine }}$
Right Intraparietal Sulcus
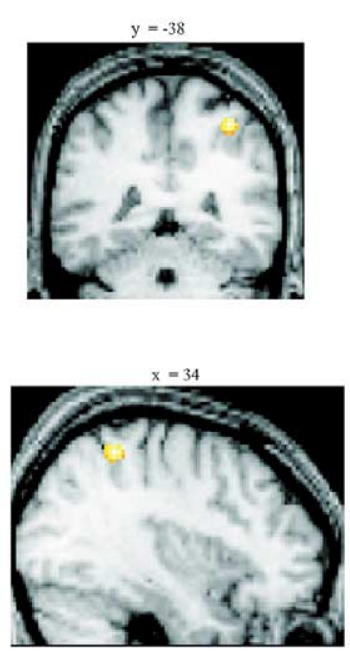

Right IPS

$34,-38,40$ $\sqcap$

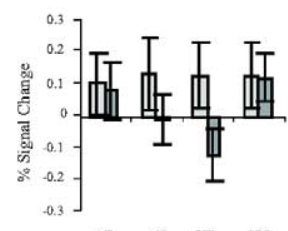

QPlacebo QPhysostigmine
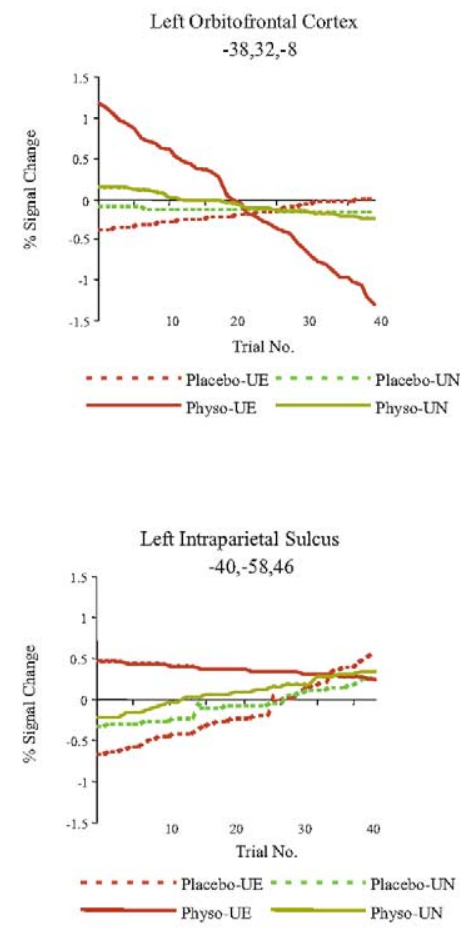
Table 1

Effects of drug on attentional modulation of face and house processing

\begin{tabular}{|c|c|c|c|c|c|}
\hline Area & & $x$ & $y$ & $z$ & $Z$ value \\
\hline \multicolumn{6}{|c|}{ (A) Attentional enhancement of face processing: increased by physostigmine } \\
\hline & Left anterior fusiform gyrus & -38 & -36 & -26 & 4.23 \\
\hline \multicolumn{6}{|c|}{ (B) Attentional enhancement of face processing: decreased by physostigmine } \\
\hline & Left insula/claustrum & -30 & -18 & 6 & 4.34 \\
\hline & Right insula & 44 & -20 & 10 & 3.57 \\
\hline \multicolumn{6}{|c|}{$\begin{array}{l}\text { (C) Attentional enhancement of house processing: increased by physostigmine } \\
\text { No areas approached significance } \\
\text { (D) Attentional enhancement of house processing } \text {. decreased by physostiomine }\end{array}$} \\
\hline (D) & $\begin{array}{l}\text { Attentional enhancement of house processing: decreased by physostigmine } \\
\text { Right lateral occipital gyrus }\end{array}$ & 38 & -88 & 4 & 4.50 \\
\hline
\end{tabular}

It has previously been shown that the effect of emotional and distracting stimuli can habituate with time (e.g., Breiter et al., 1996; Lorch et al., 1984). Consequently, the RT difference between emotional and neutral trials was calculated separately for each of the four successive blocks, with planned group comparisons made in the first block. During face-attended trials, both groups showed a trend to a timedependent effect of emotion $(F(3,27)=2.7 ; P<0.1)$, with a significant slowing of RTs to emotional stimuli within the first block only $(t(28)=2.6 ; P<0.05$; Fig. 2$)$. There were no between-group RT differences for these face-attended trials. However, during face- unattended trials, the physostigmine group showed significant slowing relative to the placebo group by the presence of an emotional expression in the first block $(t(28)=2.1 ; P<0.05$; Fig. 2). Because of these behavioral patterns, block sequence was also considered in time-related fMRI analyses.

\section{fMRI data: effects of physostigmine on attentional modulation}

We first aimed to replicate the findings from two previous studies using a similar paradigm but with untreated subjects (Wojciulik et al., 1998; Vuilleumier et al., 2001) by examining the placebo group for responses dependent on whether faces or houses fell in the task-relevant locations (i.e., attending faces minus attending houses and vice versa). As in those previous studies, we found bilateral midfusiform gyrus activation when faces appeared at task-relevant locations, while bilateral parahippocampal and posterolateral occipital cortices were activated when houses appeared at task-relevant locations (Figs. 3A and 3B, respectively; all $Z \geq 4.29 ; P<0.05$, corrected for fusiform ROIs or whole brain). These regions were also all found to be activated by the same contrasts in the physostigmine group (all $Z \geq 3.30 ; P<0.001$, uncorrected).

We next identified regions whose differential activity for faces relative to houses (or vice versa) was greater under physostigmine relative to placebo (or vice versa; Table 1). With faces versus houses in attended locations, physostigmine enhanced differential activation in left anterior fusiform gyrus (Fig. 3C) relative to placebo. This region failed to show a significant effect of face attention under placebo. The only regions showing less differential activity under drug, relative to placebo, for faces in attended versus unattended locations were bilateral insula.

Fig. 4. (A) Regions of the inferotemporal cortex showing the effect of physostigmine on emotional processing. Red represents regions in the placebo group that showed an increased response to emotional versus neutral faces, the physostigmine group can be seen to display similar effects here (A). Yellow represents regions in which physostigmine, relative to placebo, resulted in an enhanced differential response to emotional versus neutral faces (B). Both contrasts are thresholded at $P<0.01$, uncorrected (so as to illustrate less significant activation of the right fusiform in the placebo group-in the same region previously showing emotional modulation in untreated subjects: Vuilleumier et al., 2001). Activation maps are superimposed on a single-subject T1-weighted MRI brain, pitched to visualize both contrasts. Graphs represent percentages of signal change from baseline during emotional and neutral trials with faces in attended (AE and AN) or unattended (UE and UN) locations for drug and placebo groups, mean-corrected between regions. The voxels chosen are based upon the two voxels in our previous study (Vuilleumier et al., 2001) showing the most significant modulation of the fusiform cortex by attention. In the right fusiform (A), both groups show a positive main effect of emotion $(P<0.01)$; in the left fusiform, physostigmine shows a main effect of emotion $(P<0.005)$, but not placebo; a group by emotion interaction was also observed here $(P<0.005)$.

Fig. 5. Regions showing modulation of emotional responses by physostigmine selectively when faces were task-irrelevant on examination of both time-independent (A) and time-dependent (B) effects. (A) The lateral orbitofrontal and right intraparietal regions showing a significant drug by emotion by attention interaction, due to a predominant effect within face-unattended trials. Statistical maps are overlaid on a single-subject T1-weighted MRI Graphs represent percentages of signal change from baseline during face-attended emotional and neutral trials (AE and AN) and face-unattended emotional and neutral trials (UE and UN) for drug and placebo groups. The orbitofrontal cortex demonstrated a significant enhancement to task-irrelevant emotional stimuli under physostigmine only $\left({ }^{*} P<0.005\right.$ for post hoc contrast of $\left.\mathrm{UE}-\mathrm{UN}\right)$, whereas the right intraparietal sulcus demonstrated reduced activity to task-irrelevant emotional stimuli under physostigmine only ( $* * P<0.001$ for post hoc contrast of UN-UE). (B) A representative profile of activity in the lateral orbitofrontal and left intraparietal regions identified in the interaction of drug $\mathrm{x}$ emotion is shown examining condition-specific effects modeled with a linear time-dependent response function, specifically for trials when faces were task-irrelevant. Plots depict the best fitting peak canonical response over trial number of the subject from each of the placebo (dashed) and physostigmine (solid) groups showing the median effect size of task-irrelevant emotion for fearful (red) and neutral (green) trials. There were no drug by emotion interactions in these two regions for task-relevant faces. 
Table 2

Effect of drug on the occipitotemporal cortex during null trials

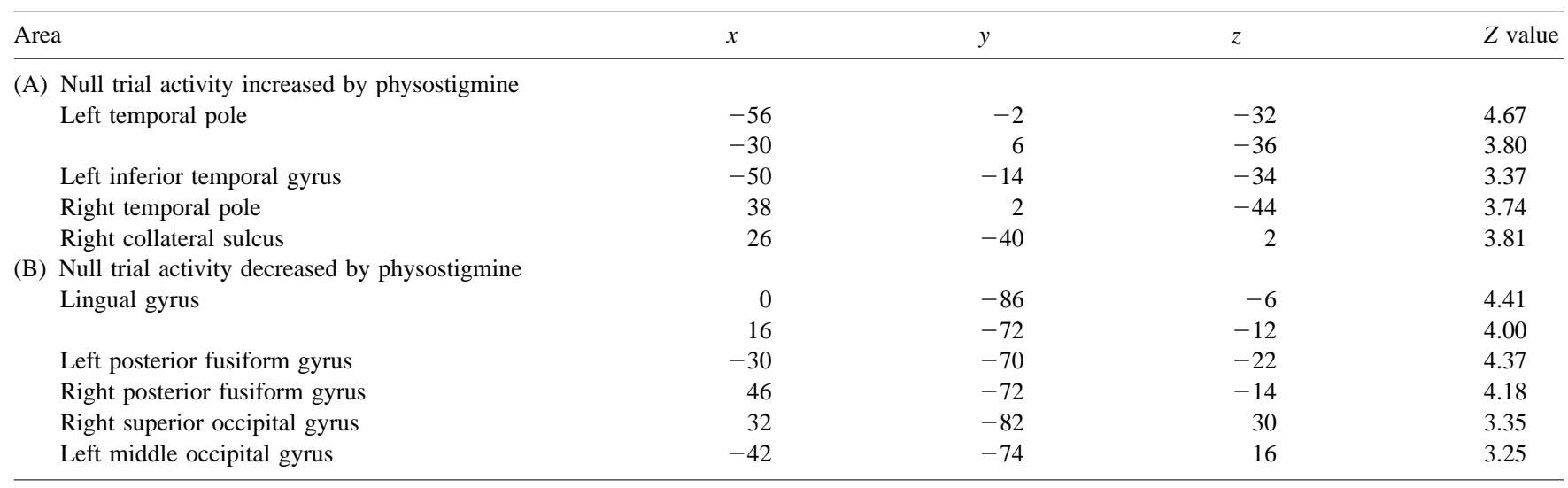

Note. Null trials represent trials in which subjects had previously been spatially cued but no stimuli appeared following an alerting central fixation cross. The mean session activity is modeled separately and was found not to be significantly different between placebo and physostigmine groups in the regions listed.

With houses versus faces in attended locations there were no enhancements of activity due to physostigmine. However, physostigmine reduced differential activity in the right posterolateral occipital cortex relative to placebo (Fig. 3D). Thus, physostigmine engendered an opposite attentional effect depending on either the stimulus-processing region or the stimulus type falling within attended locations: fusiform gyrus showed increased attentional enhancement (for attending faces minus houses), while posterolateral occipital cortex showed reduced modulation by attention (for attending houses minus faces) under physostigmine relative to placebo.

To unconfound the effect of drug on extrastriate region (fusiform and posterolateral occipital cortices) from stimulus type (faces and houses), we further tested whether the observed region-specific effects of physostigmine might correspond to a modulation of activity even in the absence of stimuli. We tested for this by comparing activations between groups in occipitotemporal regions on "null trials," when subjects were cued but no stimulus appeared (see Chawla et al., 1999). Note that these null events were modeled separately from, and hence are orthogonal to, session mean activity (thus any drug-induced changes cannot reflect overall changes in mean activity throughout a session for particular brain areas). Comparing groups, this contrast (Table 2) showed that physostigmine, relative to placebo, resulted in greater null trial activity in anteroinferior temporal regions (Fig. 3E), including left anterior fusiform, which showed the drug-by-face attention interaction described above $(t(28)=2.3, P<0.05 ;$ Fig. 3C). Conversely, physostigmine, relative to placebo, resulted in reduced activity in posterior occipital regions (Fig. 3F), although this failed to reach significance in that region showing less differential activity for house attention under physostigmine (Fig. 3D). These results indicate that the effects of physostigmine on selective attention may partly reflect regionspecific changes in activity, independently of stimulus (but related to spatial cueing), which may then either increase or decrease the differential response to attended versus unattended stimuli that are selectively processed in these regions. Furthermore, these drug-induced changes occurred only when attention was spatially cued, as shown by the fact there were no between-group differences in session means for either the inferior temporal or posterior occipital regions identified by the group-by-attention and group-by-null trial interactions.

\section{fMRI data: effects of physostigmine on response to fearful expression}

The orthogonal contrast of fearful minus neutral faces was performed, independently of whether faces were attended or unattended, on each trial. Within extrastriate cortices, the placebo group demonstrated heightened activity to fearful relative to neutral faces in left mid-fusiform cortex (-40, -48, $-24 ; Z=3.27 ; P<0.001$, uncorrected). In addition, the same voxel in right mid-fusiform cortex (44, $-52,-20)$ that showed emotion modulation in our previous study (Vuilleumier et al., 2001) demonstrated a similar effect in our data, but at a lower significance $(Z=2.64 ; P$ $<0.01$, uncorrected; Fig. 4A). These areas were also activated by emotional stimuli under physostigmine (all $Z>$ 3.09, $P<0.001$, uncorrected). The main effect of emotion over all subjects also identified a region extending between the hypothalamus and posterior medial amygdala $(10,-8$, $-16 ; Z=3.49 ; P<0.001$, uncorrected; and $12,-10,-16$; $Z=3.06 ; P<0.05$ corrected for right amygdala volume identified in Vuilleumier et al., 2001, thresholded at $P<$ 0.01 , uncorrected). Furthermore, a more lateral amygdalacentred activation was activated just below threshold (30, $-6,-18 ; Z=2.45 ; P=0.007$, uncorrected). Activity in both of these areas was independent of attention (in keeping with Vuilleumier et al., 2001) and group.

Comparing drug and placebo groups for the effect of emotion, we found regions in left middle fusiform $(-48$, $-52,-22 ; \mathrm{Z}=2.85 ; P<0.05$, corrected for ROI; Fig. 4B) and inferior occipital cortex $(-24,-94,-8 ; Z=3.37 ; P<$ 
Table 3

Areas showing effects of drug on interaction of attention with emotion

\begin{tabular}{|c|c|c|c|c|c|}
\hline \multicolumn{2}{|l|}{ Area } & \multirow[t]{2}{*}{$x$} & \multirow[t]{2}{*}{$y$} & \multirow[t]{2}{*}{$z$} & \multirow[t]{2}{*}{$Z$ value $^{\mathrm{b}}$} \\
\hline (A) $\mathrm{H}$ & Enhanced activity to task-irrelevant emotion by physostigmine & & & & \\
\hline & Left inferior temporal pole & -44 & 6 & -44 & 4.56 \\
\hline & Anterior cingulate & -2 & 18 & 22 & 3.83 \\
\hline & Left lateral orbitofrontal cortex & -30 & 40 & -16 & 3.49 \\
\hline \multicolumn{6}{|c|}{ (B) Reduction of activity to task-irrelevant $e$} \\
\hline & Right intraparietal sulcus & 34 & -38 & 40 & 4.17 \\
\hline & Left precentral gyrus & -34 & -12 & 52 & 3.39 \\
\hline & Left superior temporal sulcus ${ }^{\mathrm{a}}$ & -52 & -14 & -8 & 4.16 \\
\hline \multicolumn{6}{|c|}{ (C) Enhancement of activity to task-relevant emotion by physostigmine } \\
\hline & Left dorsolateral prefrontal cortex ${ }^{\mathrm{a}}$ & -32 & 52 & 26 & $4.90^{*}$ \\
\hline & & -40 & 30 & 18 & 4.39 \\
\hline & Medial prefrontal cortex & 6 & 46 & 26 & 3.64 \\
\hline \multicolumn{6}{|c|}{ (D) Reduction of activity to task-relevant emotion by physostigmine } \\
\hline & Ventral striatum ${ }^{\mathrm{a}}$ & -24 & 8 & -4 & 3.41 \\
\hline & Right medial orbitofrontal cortex & 22 & 48 & -8 & 3.36 \\
\hline
\end{tabular}

Note. The formal notation for the interactions referred to in the table are (A and D) physostigmine [(UE-UN)-(AE-AN] $>$ placebo [(UE-UN)-(AE-AN)]; (B and C) physostigmine [(AE-AN)-(UE-UN]>placebo [(AL-AN)-(UE-UN)].

${ }^{a}$ Areas showing significant drug $\mathrm{x}$ emotion interactions with both task-relevant and task-irrelevant faces are listed under the heading appropriate for the stronger effect.

${ }^{\text {b }}$ All $Z$ scores represent $P<0.001$, uncorrected, except* (corrected for whole brain volume).

0.001, uncorrected) that showed a greater differential response to emotional versus neutral stimuli under physostigmine, relative to placebo. Furthermore, by examining emotional effects in the extrastriate cortex that habituated with time (see Buchel et al., 1999; Morris et al., 2001; Rotshtein et al., 2001)_-in keeping with the time-dependent patterns observed behaviorally-we found that left mid-fusiform cortex also showed a stronger relative response to emotional stimuli with drug relative to placebo, as a function of time (-34, $-52,-6 ; Z=3.26 ; P<0.001$, uncorrected). There were no regions in which an emotional activation under placebo was significantly reduced by physostigmine.

\section{fMRI data: effects of physostigmine on the interaction of attention with emotion}

Finally, we examined physostigmine modulation of emotional responses as a function of whether emotional faces were task-relevant or task-irrelevant. The majority of effects were in regions previously found to exhibit an attention $\mathrm{x}$ emotion interaction (Vuilleumier et al., 2001; Armony and Dolan, 2002; Perlstein et al., 2002). Thus physostigmine versus placebo showed enhanced differential activity for emotional faces, specifically when task-irrelevant (i.e. (UE-UN)-(AE-AN)), in left lateral orbitofrontal cortex, temporal pole, and anterior cingulate, while decreasing activity in the right intraparietal sulcus for the equivalent contrast (Table 3; Fig. 5A).

Areas where physostigmine, relative to placebo, showed enhanced differential activity for emotional faces when task-relevant (i.e. (AE-AN)-(UE-UN)), faces were seen in the left dorsolateral prefrontal and medial prefrontal cortex (Table 3). The only areas where physostigmine reduced activity related to the emotion of task relevant faces were in the ventral striatum and medial orbitofrontal cortex.

To complement our RT findings of physostigmine-induced, time-dependent effects for fearful faces in task-irrelevant locations, we also examined fMRI data for an interaction of drug $\times$ task-irrelevant emotion that habituated over the course of the experiment (using a linearly decreasing time model-see Buchel et al., 1999). Results of this analysis were broadly similar to those of the time-independent fMRI effects. Thus left lateral orbitofrontal cortex $(-38,32,-8)$, adjacent inferior frontal cortex $(-44,38,6)$, right temporal pole $(48,8,-20)$, plus left intraparietal sulcus $(-40,-58,46 ; Z \geq 3.85$ for all above; Fig. 5B) showed stronger time-dependent effects for task-irrelevant emotional versus neutral faces under physostigmine relative to placebo, while left superior parietal $(-14,-56,68)$ and right occipital cortices $(32,-82,36 ; Z \geq 3.36 ; P<0.001$, uncorrected for all above) showed a reduced effect with physostigmine for the equivalent contrast.

\section{Discussion}

Cholinergic modulation of attentional effects within visual cortex

The task employed here has previously been shown in untreated subjects to activate face-related and house-related regions of extrastriate cortex differentially, depending solely on endogenous spatial attention (i.e., when both types of stimuli are presented on every trial, but with only one type falling at the currently attended locations; Vuilleumier et al., 2001; Wojciulik et al., 1998). We now show that 
physostigmine enhanced the anterior fusiform gyrus (a region linked to higher level processing of faces: George et al., 1999) for attended versus unattended faces, but suppressed differential responses in posterolateral occipital cortex for attended versus unattended houses. These results suggest that while acetylcholine can enhance the effect of selective attention within the extrastriate visual cortex, not all stimulus processing regions, or stimulus types, are affected in a similar fashion. The cortical cholinergic system may thus facilitate selective attention not only via general influences on "top-down" processes within frontoparietal cortices (Sarter et al., 2001; Himmelheber et al., 2001)which would predict parallel effects for face and houseselective regions-but also through region-specific effects in sensory perceptual areas.

To distinguish whether these distinct effects of physostigmine on attentional processing were a function of extrastriate cortical region (fusiform or posterolateral occipital cortex) or stimulus type attended (faces or houses), we examined activity in these same regions on null trials, when subjects were cued but no stimulus appeared. This showed that event-related activity in anterior fusiform cortex was enhanced, while that in occipital cortex was decreased by physostigmine, even in stimulus absence. Thus, drug-induced changes on null trials occurred in the same direction as when a stimulus was present and were associated with similar changes in the differential responses caused by attention. This may suggest that physostigmine modified the general responsiveness of extrastriate cortex according to region, rather than stimulus type. Importantly, these druginduced regional modulations were observed only to the event related responses to cued trials and were not seen as group differences in baseline activity.

It is noteworthy that similar regional effects of cholinergic modulation have been found in previous functional imaging studies, across a wide variety of tasks. Thus while posterolateral occipital areas have been associated with cholinergic-induced activity decreases (Mentis et al., 2001; Grasby et al., 1995; Thiel et al., 2001), fusiform cortex has been associated with cholinergic-induced increases (Furey et al., 1997, 2000a; Rosier et al., 1999; Thiel et al., 2002b; some of these studies employed cholinergic antagonists to demonstrate the converse effects). Our data extend these findings by showing regional differences in cholinergic modulation for effects of selective attention, when stimuli and task are kept constant.

A further interpretation of the extrastriate region-specific effects of physostigmine observed here was that they were dependent on the stimuli expected. Hence cholinergic enhancement may have biased activations in advance of any stimulation (see Chawla et al., 1999) to favor enhancements of face attention (in fusiform) and reductions of house attention effects (in posterolateral occipital cortex). However, any biasing cannot have taken the extreme form of the physostigmine group always attending to the faces, regardless of whether these were task-relevant. This could only have reduced differential activation for task-relevant versus irrelevant faces in the fusiform cortex, whereas in fact physostigmine either enhanced this effect (left anterior fusiform) or did not diminish it (bilateral mid-fusiform). Thus, in terms of brain responses, top-down selection continued to operate under physostigmine, but with task-relevant faces becoming particularly dominant, in keeping with the anteroinferior temporal activations.

Neuropharmacological studies have found that acetylcholine can result in differing relative levels of activation versus inhibition in the visual cortex depending on local factors (e.g., Xiang et al., 1998; Muller and Singer, 1989; Sillito and Kemp, 1983). Within the inferior temporal cortex, cholinergic stimulation has been proposed to underlie the diffuse activation seen at the start of new delayedmatch-to-sample trials when attention is heightened (Sohal and Hasselmo, 2000; Furey et al., 2000a). Furthermore, the anteroinferior temporal cortex represents a unique sensory region in having projections both to and from the nucleus basalis (Mesulam and Mufson, 1984) and so may influence its own activation through a cholinergic-dependent feedback loop (Sohal and Hasselmo, 2000). Conversely, early visual cortical areas have been found to be inhibited by acetylcholine in all layers except layer IV (Kimura et al., 1999), which may favor feedforward over feedback activity (Hasselmo, 1995) and enhance direction and orientation specificities (Murphy and Sillito, 1991). Consequently, the contrasting activity profile between inferior temporal and occipital cortices observed here in response to systemically administered anticholinesterase may reflect such regional differences of net neural activation versus inhibition. Moreover, our finding that such changes in activity were trial specific may relate to the fact that endogenous cholinergic release elevates with attentional demand (Gill et al., 2000).

\section{Cholinergic modulation of emotional effects within visual cortex}

Corticopetal cholinergic fibers appear to be involved in both filtering out distractors (Gill et al., 2000), consistent with the modulation of attentional effects reported here, and enhancing responses to stimuli of emotional significance (Acquas et al., 1996). One mechanism for this may involve direct cholinergic modulation of the visual cortex, similar to that found in the auditory (Weinberger et al., 1998) and somatosensory cortices (Delacour et al., 1990) during conditioning in rodents. Moreover, connections between the amygdala, nucleus basalis, and sensory cortical regions may provide one pathway (Amaral et al., 1992) by which emotional discriminations within the amygdala can facilitate relevant stimulus processing (Wilson and Rolls, 1990; Kapp et al., 1990; Weinberger et al., 1990; Friston et al., 1994; Morris et al., 2001).

The results of our placebo group, and those of a previous report employing the present study design (without drug: Vuilleumier et al., 2001) found that the right mid-fusiform 
gyrus was modulated by both attention and emotion separately (i.e., additively; Fig. 4A). We now demonstrate that cholinergic enhancement can increase the extent of emotional modulation within the left mid-fusiform gyrus (Fig. 4B), as well as the posterior occipital cortex. As with the enhancement of face attention discussed earlier, the left laterality of this drug effect may arise because the right fusiform is already highly sensitive to faces and their emotion, while the left fusiform becomes more so with cholinergic enhancement. The fact that left fusiform cortex also showed enhanced responses to emotional stimuli when we modeled time habituating effects (akin to those seen in behavior; see also Buchel et al., 1999; Breiter et al., 1996; Morris et al., 2001; Rotshtein et al., 2001) is consistent with cell recordings in the substantia innominata suggesting that cortical cholinergic stimulation occurs selectively with novel, behaviorally relevant stimuli (Wilson and Rolls, 1990).

Finally, we note that in addition to enhancing fusiform gyrus activity, the main effect of fearful versus neutral faces activated a region around the right hypothalamus-dorsomedial amygdala, with only a trend for activation centred on the right amygdala proper. In this respect, our findings in the placebo group did not entirely replicate those in our earlier study (Vuilleumier et al., 2001). However, this difference might be attributable to a change in several parameters, including stimulus set, number of events, interstimulus interval, statistical analysis (see Materials and methods), as well as the stress of venipuncture and infusion. We note that the two amygdala-related regions showing fear-related activations were unaffected by either attention (as in Vuilleumier et al., 2001) or drug (consistent with the amygdala being upstream of nucleus basalis activation).

\section{Cholinergic modulation of attention-emotion interaction in the frontoparietal cortex}

Cholinergic projections from the nucleus basalis to the frontoparietal cortex and thalamus may provide a means by which emotional processing engages attention (Holland and Gallagher, 1999; Friston et al., 1994); when overactive, this may contribute to clinical anxiety (Bernston et al., 1998; Hart et al., 1999). Previous functional imaging studies have identified distinct frontoparietal regions that respond to emotional stimuli in a manner that depends on the degree to which the stimuli are task-relevant (Vuilleumier et al., 2001; Armony and Dolan, 2002; Perlstein et al., 2002). By examining areas that showed an interaction of attention by emotion by drug, we found that many of these regions also displayed a cholinergic-induced modulation of responses to emotional faces that depended on whether the faces were task relevant.

Physostigmine, relative to placebo, resulted in an enhancement to task-irrelevant fearful faces in the lateral orbitofrontal cortex (OFC), anterior cingulate, and temporal pole, whereas the right intraparietal sulcus showed a decre- ment in response for the equivalent contrast (Fig. 5A). The lateral OFC and left intraparietal sulcus also showed physostigmine-specific enhancements to task-irrelevant fearful faces that decreased with time (Fig. 5B), in line with a parallel impairment in RTs under physostigmine that was similarly time-dependent (Fig. 2). These data support the view (Armony and Dolan, 2002; Elliot et al, 2000) that this network of areas relays information about the affective value of background stimuli to attentional processes and, furthermore, they show an increase in this effect with cholinergic enhancement. Animal studies have previously shown that the OFC is intimately connected with cholinergic fibers of the nucleus basalis (Cavada et al., 2000) and is activated by acetylcholine during reinforcement (Aou et al., 1983), while cholinergic modulation of the parietal cortex influences covert spatial attention (Davidson and Marrocco; 2000), including that toward reward-associated stimuli (Chiba et al., 1995; Bucci et al., 1998). Here we have demonstrated that cholinergic enhancement both modulated activity in the OFC and parietal cortex, and resulted in impairment of performance, specifically under the condition of task-irrelevant fearful faces (in a time-dependent manner for both).

By contrast, physostigmine increased responses to taskrelevant fearful faces in dorsolateral and medial prefrontal cortices, which have previously shown emotion-sensitive task-relevant activity (e.g., Simpson et al., 2000; Gray et al., 2002; Perlstein et al., 2002). These regions have also been found to depend on cholinergic afferents for both selective attention (Muir et al., 1996; Gill et al., 2000) and enhancement of cortical responses to emotional stimuli (Mark et al., 1996; Acquas et al., 1996; Thiel et al., 1998; Pirch et al., 1992). The pattern of activity in prefrontal areas under physostigmine seen here is particularly in keeping with a model of anxiety which proposes excessive cholinergic stimulation of the prefrontal cortex as a means by which fearful stimuli are processed excessively (see Hart et al., 1999; Bernston et al., 1998). In contrast to the case with task-irrelevant emotional stimuli, RTs were impaired with task-relevant emotional stimuli to a similar extent under physostigmine and placebo, suggesting a ceiling effect in placebo.

\section{Conclusion}

Our study has shown that neural correlates of both selective attention and emotional processing can be independently enhanced by physostigmine in the fusiform gyrus. By contrast, physostigmine decreased differential activation due to attention in the posterolateral occipital cortex. As these changes occurred even in the absence of stimuli we suggest that acetylcholine may modulate attention according to extrastriate region, rather than stimulus type. Physostigmine also modulated responses to emotional stimuli depending on whether they were task-irrelevant (in orbitofrontal and intraparietal cortices) or task-relevant (in dorso- 
lateral and medial prefrontal cortices). These results demonstrate that despite their diffuse neocortical innervation, cholinergic projections may modulate attention-related and emotion-related activity in distinct parts of extrastriate and frontoparietal cortices.

\section{Acknowledgments}

This work was supported by a program grant from the Wellcome Trust to R.J.D.; C.M.T receives a research grant from the German Research Foundation (Deutsche Forschungsgememschaft), J.D. holds a Royal Society-Wolfson Research Merit Award. We thank J. Ashburner, J. Winston, and I. Benton from Wardray Premise.

\section{References}

Acquas, E., Wilson, C., Fibiger, H.C., 1996. Conditioned and unconditioned stimuli increase frontal cortical and hippocampal acetylcholine release: effects of novelty, habituation, and fear. J. Neurosci. 16, 3089-3096.

Amaral, D.G., Price, J.L., Pitkanen, A., Carmichael, S.T., 1992. Anatomical organization of the primate amygdaloid complex, in: Aggleton, J.P. (Ed.), The Amygdala: Neurobiological Aspects of Emotion, Memory, and Mental Dysfunction. Wiley-Liss, New York, pp. 1-66.

Aou, S., Oomura, Y., Nishino, H., 1983. Influence of acetylcholine on neuronal activity in monkey orbitofrontal cortex during bar press feeding task. Brain Res. 275, 178-182.

Armony, J.L., Dolan, R.J., 2002. Modulation of spatial attention by fearconditioned stimuli: an event-related fMRI study. Neuropsychologia 40, 817-826.

Ballard, C., O'Brien, J, Gray, A., et al., 2001. Attention and fluctuating attention in patients with dementia with Lewy bodies and Alzheimer disease. Arch. Neurol. 58, 977-982.

Bentley P, Vuilleumier P, Thiel C.M. Driver J. Dolan R.J., 2003. Effects of attention and emotion on repetition priming and their modulation by cholinergic enhancement. J. Neurophysiol., (in press).

Berntson, G.G., Sarter, M., Cacioppo, J.T., 1998. Anxiety and cardiovascular reactivity: the basal forebrain cholinergic link. Behav. Brain Res. 94, 225-248.

Bond, A., Lader, M., 1974. The use of analogue scales in rating subjective feelings. Br. J. Med. Psychol. 47, 211-218.

Breiter, H.C., Etcoff, N.L., Whalen, P.J., et al., 1996. Response and habituation of the human amygdala during visual processing of facial expression. Neuron 17, 875-887.

Bucci, D.J., Holland, P.C., Gallagher, M., 1998. Removal of cholinergic input to rat posterior parietal cortex disrupts incremental processing of conditioned stimuli. J. Neurosci. 18, 8038-8046.

Buchel, C., Dolan, R.J., Armony, J.L., Friston, K.J., 1999. Amygdalahippocampal involvement in human aversive trace conditioning revealed through event-related functional magnetic resonance imaging. J. Neurosci. 19, 10869-10876.

Cavada, C., Company, T., Tejedor, J., Cruz-Rizzolo, R.J., Reinoso-Suarez, F., 2000. The anatomical connections of the macaque monkey orbitofrontal cortex. A review. Cereb. Cortex 10, 220-242.

Chawla, D., Rees, G., Friston, K.J., 1999. The physiological basis of attentional modulation in extrastriate visual areas. Nat. Neurosci. 2, 671-676.

Chiba, A.A., Bucci, D.J., Holland, P.C., Gallagher, M., 1995. Basal forebrain cholinergic lesions disrupt increments but not decrements in conditioned stimulus processing. J. Neurosci. 15, 7315-7322.
Davidson, M.C., Marrocco, R.T., 2000. Local infusion of scopolamine into intraparietal cortex slows covert orienting in rhesus monkeys. J. Neurophysiol. 83, 1536-1549.

Delacour, J., Houcine, O., Costa, J.C., 1990. Evidence for a cholinergic mechanism of "learned" changes in the responses of barrel field neurons of the awake and undrugged rat. Neuroscience 34, 1-8.

Elliott, R., Dolan, R.J., Frith, C.D., 2000. Dissociable functions in the medial and lateral orbitofrontal cortex: evidence from human neuroimaging studies. Cereb. Cortex 10, 308-317.

Friston, K.J., Tononi, G., Reeke Jr., G.N., Sporns, O., Edelman, G.M., 1994. Value-dependent selection in the brain: simulation in a synthetic neural model. Neuroscience 59, 229-243.

Friston, K.J., Holmes, A.P., Worsley, K.J., Poline, J.B., Frith, C.D., Frackowiak, R.S.J., 1995. Statistical parametric maps in functional imaging: A general linear approach. Hum. Brain Mapp. 2, 189-210.

Furey, M.L., Pietrini, P., Haxby, J.V., et al., 1997. Cholinergic stimulation alters performance and task-specific regional cerebral blood flow during working memory. Proc. Natl. Acad. Sci. USA 94, 6512-6516.

Furey, M.L., Pietrini, P., Haxby, J.V., 2000a. Cholinergic-enhancement and increased selectivity of perceptual processing during working memory. Science 290, 2315-2319.

Furey, M.L., Pietrini, P., Alexander, G.E., et al., 2000b. Time course of pharmacodynamic and pharmacokinetic effects of physostigmine assessed by functional brain imaging in humans. Pharmacol. Biochem. Behav. 66, 475-481.

George, N., Dolan, R.J., Fink, G.R., Baylis, G.C., Russell, C., Driver, J., 1999. Contrast polarity and face recognition in the human fusiform gyrus. Nat. Neurosci. 2, 574-580.

Gill, T.M., Sarter, M., Givens, B., 2000. Sustained visual attention performance-associated [medial] prefrontal neuronal activity: evidence for cholinergic modulation. J. Neurosci. 20, 4745-4757.

Grasby, P.M., Frith, C.D., Paulesu, E., Friston, K.J., Frackowiak, R.S., Dolan, R.J., 1995. The effect of the muscarinic antagonist scopolamine on regional cerebral blood flow during the performance of a memory task. Exp. Brain Res. 104, 337-348.

Gray, J.R., Braver, T.S., Raichle, M.E., 2002. Integration of emotion and cognition in the lateral prefrontal cortex. Proc. Natl. Acad. Sci USA 99. $4115-4120$

Hart, S., Sarter, M., Berntson, G.G., 1999. Cholinergic inputs to the rat medial prefrontal cortex mediate potentiation of the cardiovascular defensive response by the anxiogenic benzodiazephine receptor partial inverse agonist FG 7142. Neuroscience 94, 1029-1038.

Hasselmo, M.E., 1995. Neuromodulation and cortical function modeling the physiological basis of behavior. Behav. Brain Res. 67, 1-27.

Himmelheber, A.M., Sarter, M., Bruno, J.P., 2001. The effects of manipulations of attentional demand on cortical acetylcholine release. Brain Res. Cogn. Brain Res. 12, 353-370.

Holland, P.C., Gallagher, M., 1999. Amygdala circuitry in attentional and representational processes. Trends Cogn. Sci. 3, 65-73.

Kapp, B.S., Wilson, A., Pascoe, J.P., Supple, W., Whalen, P.J., 1990. A neuroanatomical systems analysis of conditioned bradycardia in the rabbit, in: Gabriel, M., Moore, J. (Eds.), Learning and Computational Neuroscience: Foundations of Adaptive Networks. MIT Press, Cambridge, MA, pp. 53-90.

Kimura, F., Fukuda, M., Tsumoto, T., 1999. Acetylcholine suppresses the spread of excitation in the visual cortex revealed by optical recording: possible differential effect depending on the source of input. Eur. J. Neurosci. 11, 3597-3609.

Lawrence, A.D., Sahakian, B.J., 1995. Alzheimer disease, attention, and the cholinergic system. Alzheimer Dis. Assoc. Disord. 9 (Suppl. 2), 43-49.

Lawrence, S.L., Ross, T.J., Stein, E.A., 2002. Cognitive mechanisms of nicotine on visual attention. Neuron 36, 539-548

Lorch, E.P., Anderson, D.R., Well, A.D., 1984. Effects of irrelevant information on speeded classification tasks: Interference is reduced by habituation. J. Exp. Psychol: Hum. Perception Performance 10, 850864. 
Lundqvist, D., Flykt, A., Vhman, A., 1998. The Karolinska Directed Emotional Faces. Pictoral face set available from the Department of Neurosciences, Karolinska Hospital, Stockholm, Sweden.

Mark, G.P., Rada, P.V., Shors, T.J., 1996. Inescapable stress enhances extracellular acetylcholine in the rat hippocampus and prefrontal cortex but not the nucleus accumbens or amygdala. Neuroscience 74, 767774.

McGaughy, J., Kaiser, T., Sarter, M., 1996. Behavioral vigilance following infusions of $192 \mathrm{IgG}$-saporin into the basal forebrain: selectivity of the behavioral impairment and relation to cortical AChE-positive fiber density. Behav. Neurosci. 110, 247-265.

McKeith, I., Del Ser, T., Spano, P., et al., 2000. Efficacy of rivastigmine in dementia with Lewy bodies: a randomised, double-blind, placebocontrolled international study. Lancet 356, 2031-2036.

Mentis, M.J., Sunderland, T., Lai, J., et al., 2001. Muscarinic versus nicotinic modulation of a visual task. a pet study using drug probes. Neuropsychopharmacology 25, 555-564.

Mesulam, M.M., Mufson, E.J., 1984. Neural inputs into the nucleus basalis of the substantia innominata (Ch4) in the rhesus monkey. Brain 107 (Pt. 1), 253-274.

Morris, J.S., Buchel, C., Dolan, R.J., 2001. Parallel neural responses in amygdala subregions and sensory cortex during implicit fear conditioning. Neuroimage 13, 1044-1052.

Muir, J.L., Everitt, B.J., Robbins, T.W., 1996. The cerebral cortex of the rat and visual attentional function: dissociable effects of mediofrontal, cingulate, anterior dorsolateral, and parietal cortex lesions on a fivechoice serial reaction time task. Cereb.Cortex 6, 470-481.

Muller, C.M., Singer, W., 1989. Acetylcholine-induced inhibition in the cat visual cortex is mediated b7 a GABAergic mechanism. Brain Res. 487 335-342.

Murphy, P.C., Sillito, A.M., 1991. Cholinergic enhancement of direction selectivity in the visual cortex of the cat. Neuroscience 40, 13-20.

Perlstein, W.M., Elbert, T., Stenger, V.A., 2002. Dissociation in human prefrontal cortex of affective influences on working memory-related activity. Proc. Natl. Acad. Sci. USA 99, 1736-1741.

Perry, R.J., Hodges, J.R., 1999. Attention and executive deficits in Alzheimer's disease. A critical review. Brain 122 (Pt. 3), 383-404.

Pirch, J.H., Turco, K., Rucker, H.K., 1992. A role for acetylcholine in conditioning-related responses of rat frontal cortex neurons: microiontophoretic evidence. Brain Res. 586, 19-26.

Robbins, T.W., 1998. Arousal and attention psychopharmacological and neuropsychological studies in experimental animals. in Parsuraman, $\mathrm{R}$ (Ed.), The Attentive Brain. MIT Press, Cambridge, pp. 189-220.

Rosier, A.M., Cornette, L., Dupont, P., Bormans, G., Mortelmans, L., Orban, G.A., 1999. Regional brain activity during shape recognition impaired by a scopolamine challenge to encoding. Eur. J. Neurosci. 11, 3701-3714.

Rotshtein, P., Malach, R., Hadar, U., Graif, M., Hendler, T., 2001. Feeling or features: different sensitivity to emotion in high-order visual-cortex and amygdala. Neuron 32, 747-757.
Sarter, M., Givens, B., Bruno, J.P., 2001. The cognitive neuroscience of sustained attention: where top-down meets bottom-up. Brain Res. Brain Res. Rev. 35, 146-160.

Sato, H., Hata, Y., Masui, H., Tsumoto, T., 1987. A functional role of cholinergic innervation to neurons in the cat visual cortex. J. Neurophysiol. 58, 765-780.

Sillito, A.M., Kemp, J.A., 1983. Cholinergic modulation of the functional organization of the cat visual cortex. Brain Res. 289, 143-155.

Simpson, J.R., Ongur, D., Akbudak, E., et al., 2000. The emotional modulation of cognitive processing: an fMRI study. J. Cogn. Neurosci. 12 (Suppl. 2), 157-170.

Sohal, V.S., Hasselmo, M.E., 2000. A model for experience-dependent changes in the responses of inferotemporal neurons. Network 11, 169-190.

Thiel, C.M., Huston, J.P., Schwarting, R.K., 1998. Cholinergic activation in frontal cortex and nucleus accumbens related to basic behavioral manipulations: handling, and the role of post-handling experience. Brain Res. 812, 121-132.

Thiel, C.M., Henson, R.N., Morris, J.S., Friston, K.J., Dolan, R.J., 2001. Pharmacological modulation of behavioral and neuronal correlates of repetition priming. J. Neurosci. 21, 6846-6852.

Thiel, C.M., Friston, K.J., Dolan, R.J., 2002a. Cholinergic modulation of experience-dependent plasticity in human auditory cortex. Neuron 35, 567-574.

Thiel, C.M., Henson, R.N., Dolan, R.J., 2002b. Scopolamine but not lorazepam modulates face repetition priming: a psychopharmacological fMRI study. Neuropsychopharmacology 27, 282-292.

Vuilleumier, P., Armony, J.L., Driver, J., Dolan, R.J., 2001. Effects of attention and emotion on face processing in the human brain: an event-related fMRI study. Neuron 30, 829-841.

Weinberger, N.M., Ashe, J.H., Metherate, R., McKenna, T.M., Diamond, D.M., Bakin, J.S., Lennartz, R.C., Cassady, J.M., 1990. Neural adaptive information processing: A preliminary model of receptive-field plasticity in auditory cortex during Pavlovian conditioning, in: Gabriel, M., Moore, J. (Eds.), Learning and Computational Neuroscience: Foundations of Adaptive Networks. MIT Press, Cambridge, MA, pp. 91-138.

Weinberger, N.M., 1998. Physiological memory in primary auditory cortex: characteristics and mechanisms. Neurobiol. Learn. Mem. 70, 226251.

Wilson, F.A., Rolls, E.T., 1990. Neuronal responses related to the novelty and familarity of visual stimuli in the substantia innominata, diagonal band of Broca and periventricular region of the primate basal forebrain. Exp. Brain Res. 80, 104-120.

Wojciulik, E., Kanwisher, N., Driver, J., 1998. Covert visual attention modulates face-specific activity in the human fusiform gyrus. fMRI study. J. Neurophysiol. 79, 1574-1578.

Worsley, K.J., Marrett, S., Neelin, P., Vandal, A.C., Friston, K.J., Evans, A.C., 1996. A unified statistical approach to determining significant signals in images of cerebral activation. Hum. Brain Mapp. 4, 58-73.

Xiang, Z., Huguenard, J.R., Prince, D.A., 1998. Cholinergic switching within neocortical inhibitory networks. Science 281, 985-988. 